\title{
Entanglement of 9-qubit graph states
}

\author{
Sui yan \\ College of Information and Electronic Engineering \\ Zhejiang Gongshang University \\ Hangzhou, China \\ kathyissy@163.com
}

\author{
Xiao-yu Chen \\ College of Information and Electronic Engineering \\ Zhejiang Gongshang University \\ Hangzhou, China \\ xychen@mail.zjgsu.edu.cn
}

\begin{abstract}
The entanglement of graph states can be calculated by adopting Iterative algorithm. The number of inequivalent classes of 9-qubit graph states is $\mathbf{4 4 0}$. All 440 local inequivalent graphs are classified as two categories: graphs with equal upper LOCC entanglement bound and lower bipartite entanglement bound, graphs with unequal bounds. The later may display non-integer entanglement. After determining the entanglement, the closest product state can also be obtained. As for graph states with different entanglement values, the closest product states have different structural characteristics. The precision of iteration algorithm of the entanglement is less than $10^{-14}$.
\end{abstract}

Keywords-entanglement; graph states; iterative algorithm; closest product state

\section{INTRODUCTION}

The multipartite entanglement plays an important role in quantum error correction and quantum computation. Graph states are certain pure multipartite quantum states associated to graphs [1][2][3] and have important applications in quantum error correction[4] and one-way quantum computer[5]. However, the quantification of multipartite entanglement is still open even for a pure multipartite state. Until now, a variety of different entanglement measures have been proposed for multipartite quantum states, such as, the (Global) Robustness of Entanglement [6], the Relative Entropy of Entanglement [7][8] and the Geometric Measure [9]. Nevertheless, these measures are very difficult as they are always defined as the solutions to difficult variational problems. Fortunately, these entanglement measures are all equal for stabilizer state [10]. It is known that the graph state is a subset of stabilizer state. Thus these entanglement measures are all equal for graph state.

The quantification of graph state entanglement is relatively simple. In [11], the author gives the upper and lower bounds to the entanglement of any graph state by using a simple graphical interpretation of these states, but not give the exact entanglement values of the graph states which have unequal bounds, in order to calculate the exact entanglement values, an Iterative algorithm [12] has been proposed. In [12], the author gives the exact entanglement values of graph states up to 8qubit. This paper will base on [12], calculate the entanglement of 9-qubit graph states by adopting Iterative algorithm.

The paper is organized as follows, in section II, we recall the notion of graph state, and its related concepts, the bounds of the entanglement, and describe the iteration method. Then in section III we classify all the inequivalent 9-qubit graph states, propose the precise values of entanglement and give the closest product state. Section IV is devoted to analyze the precision of the iterative algorithm. We finish with conclusions.

\section{PRELIMINARY}

\section{A. Graph state}

Graph state is associated with connected mathematical graph. A graph $G=(V ; \Gamma)$ is composed of a set $V$ of n vertices and a set of edges specified by the adjacency matrix $\Gamma$, which is an $n \times n$ symmetric matrix with vanishing diagonal entries and $\Gamma_{a b}=1$ if vertices $a, b$ are connected and $\Gamma_{a b}=0$ otherwise. The neighborhood of a vertex $a$ is the set of all the vertices that are connected to $a$, can be denoted by $N_{a}=\left\{v \in V \mid \Gamma_{a v}=1\right\}$. To associate the graph state to the underlying graph, we assign each vertex with a qubit, each edge represents the interaction between the corresponding two qubits. We denote the Pauli matrices at the $a$-th qubit by $X_{a}, Y_{a}, Z_{a}$ and identity by $I_{a}$. The graph state related to graph $G$ is defined as

$$
|G\rangle=\prod_{\Gamma_{a b}} U_{a b}|+\rangle_{x}^{V} \frac{1}{\sqrt{2^{n}}} \sum_{\mu=0}^{1}(-1)^{\frac{1}{2} \mu \Gamma \mu^{T}}|\mu\rangle_{Z}
$$

where $\left|\mu_{z}\right\rangle$ is the joint eigenstate of Pauli operators $Z_{a}(a \in V)$ with eigenvalues $(-1)^{\mu_{a}},|+\rangle_{x}$ is the joint +1 eigenstate of Pauli operators $X_{a}(a \in V)$, and $U_{a b}$ is the controlled phase gate between qubits $a$ and $b$.

A widely used local operation in dealing with graph states is the local complementation (LC) [13], which leaves the entanglement properties invariant. LC centered on a qubit $a$ is visualized as a transformation of the subgraph of $a$-th qubit's neighbours. Graphically, LC on the qubit $a$ acts as follows: One picks out the vertex $a$ and inverts the neighborhood $N(a)$, i.e. vertices in the neighborhood which were connected become disconnected and vice versa.

\section{B. Entanglement bounds}

The entanglement is upper bounded by the local operation and classical communication ( $L O C C$ ) bound $E_{L O C C}$, and lower 
bounded by some bipartite entanglement deduced from the state, i.e. the "matching" bound $E_{b i}$ [11]. The inequality for the entanglement of a graph state is:

$$
E_{b i} \leq E \leq E_{\text {LOCC }}
$$

If the lower bound coincides with the upper bound, the entanglement of the graph state can be obtained. The upper $L O C C$ bound for a graph state is:

$$
E \leq n-|A|
$$

Where $|\mathrm{A}|$ is the largest number of vertices which any two of them being not adjacent [11]. The entanglement is lower bounded by the entanglement of a bipartition of the graph. The lower bound can be found by "matching" [11]. A convenient way of finding the lower bound of the entanglement is to find the largest set of nonadjacent edges [12].

\section{Iterative algorithm}

If the lower bound and the upper bound do not meet, we will use a systematical Iterative algorithm [12] to calculate the entanglement of such graph states.

For a graph state $|G\rangle$, the measures of entanglement are equal, and entanglement can be written as

$$
E(|G\rangle)=\min _{\phi \in \operatorname{Pr} o}-\log _{2}|\langle G \mid \phi\rangle|^{2}=-\log _{2}\left(\max _{\phi \in \operatorname{Pr} o} F_{\phi}\right)
$$

Where $|\phi\rangle=\prod\left(\sqrt{p_{j}}|0\rangle+\sqrt{1-p_{j}} e^{i \nu}|1\rangle\right)$ is the product pure state, $F_{\phi}=|\langle G \mid \phi\rangle|^{2}$ is the fidelity between graph state and the product pure state. Iterative equation is derived from maximizing $F_{\phi}$, that is, to find the closest product state.

The product pure state $|\phi\rangle$ can also be denoted as

$$
|\phi\rangle=\prod_{j}\left(x_{j}|0\rangle+y_{j}|1\rangle\right)
$$

Where $x_{j}$ and $y_{j}$ are complex numbers subjected to $\left|x_{j}\right|^{2}+\left|y_{j}\right|^{2}=1$.

Denote $f=\langle G \mid \phi\rangle$, then

$$
f=\frac{1}{\sqrt{2^{n}}} \sum_{0}^{1}(-1)^{\frac{1}{2} \mu \Gamma \mu^{T}} \prod_{j}\left(x_{j}^{1-\mu_{j}} y_{j}^{\mu_{j}}\right) .
$$

Calculation of entanglement is equivalent to maximize $f$, we use Lagrange multiplier algorithm to calculate the extreme, by calculation, the iterative equations are

$$
z_{j}^{*}=\frac{\sum_{v^{\prime}=0^{\prime}}^{1}(-1)^{\frac{1}{2} v^{\prime} \Gamma v^{\prime T}} \prod_{k \neq j} z_{k}^{\mu_{k}}}{\sum_{v=0}^{1}(-1)^{\frac{1}{2} \Gamma \Gamma v^{T}} \prod_{k \neq j} z_{k}^{\mu_{k}}}
$$

Where $z_{j}=\frac{y_{j}}{x_{j}}, 1^{\prime}=\{1, \ldots, 1,0,1, \ldots, 1\}, 0^{\prime}=\{0, \ldots, 0,1,0, \ldots 0\}$.

In each step of the iteration, the fidelity does not decrease [12]. Starting with any initial complex random vector $z=\left(z_{1}, \ldots, z_{n}\right)$ the iterative equations renew each $z_{j}$ successively, the fidelity increases until it does not increase any more, this may be the case that the fidelity reaches its local maximum. In order to find the global maximum, we run the iterative algorithm many times with random initial $\mathrm{z}$. After iterating the value of $z$, we can get $x_{j}$ and $y_{j}$, according to Eq.(6) we can calculate $f$, from Eq.(4) we can get the entanglement of graph state.

\section{ClASSIFICATION OF THE GRAPH STATES UP TO 9-QUBIT}

The graphs correspond to the 9-qubit graph states have been plotted by L.E.Danielsen with the program nauty[14]. The number of LC inequivalent graphs up to 9-qubit is 440[15], and we number the graph from No 9.1 to No 9.440 . These graphs can be classified as two categories: Graphs with identical upper LOCC entanglement bound and lower bipartite entanglement bound, and graphs with unequal bounds.

\section{A. Graph state with equal bound}

The graph states whose entanglement with equal lower and upper bounds are listed in Table I. The entanglement of these graph states can be calculated with the methods in Ref [11], i.e. the entanglement can be determined by confirming upper and lower bounds, and we use program to determine the bounds.

TABLE I. GRAPH STATES WITH EQUAL BOUND

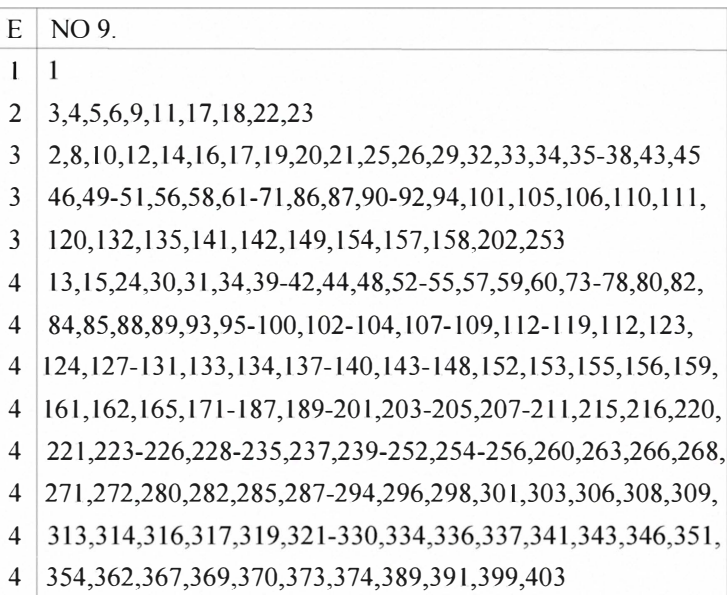




\section{B. Graph state with unequal bound}

For the other graph states, we can not determine the entanglement from the upper and lower bounds, but we can utilize Eq.(7) to find the closest product state with random initial complex numbers for $z_{j}(j=1, \ldots, n)$, and then calculate the entanglement. The values of entanglement are listed in Table II and Table III. The entanglement of graph state is integer or noninteger.

After determining the entanglement, the closest product state can also be obtained. From calculating the closest product states of graph states, we find that ring 5 graph is essential to all these graph states with entanglement $\mathrm{k}+0.9275$ (integer $\mathrm{k}$ ) and can be considered as the subgraph, so the closest product state of these graph state have a substructure of the closest product state of ring 5 graph. Closest product state is supposed for ring 5 graph state [16], and it has been shown that the entanglement of ring 5 graph state is

$$
E_{\text {ring } 5}=1+\log _{2} 3+\log _{2}(3-\sqrt{3}) \approx 2.9275
$$

Denote product state $\left|\phi_{j}\right\rangle=\sqrt{p}|0\rangle+\sqrt{1-p} e^{j \varphi_{j}}|1\rangle,(j=1, \ldots, 4)$

Where $\sqrt{p}=\sqrt{\frac{1}{2}\left(1-\frac{1}{\sqrt{3}}\right)} \approx 0.4597, \varphi_{1}=\frac{\pi}{4}, \varphi_{2}=-\frac{\pi}{4}, \varphi_{3}=\frac{3 \pi}{4}$, $\varphi_{4}=-\frac{3 \pi}{4}$. The closest product state of ring 5 graph state is .

$$
\left|\phi_{\text {ring } 5}\right\rangle=\left|\phi_{1}\right\rangle^{\otimes 5}
$$

The graph state set with noninteger entanglement value $(\mathrm{k}+0.9275)$ is specified by ring 5 graph state, and the components of the closest product states can be divided into three kinds, such as $| \pm\rangle,\left|\phi_{j}\right\rangle,|0\rangle$ or $|1\rangle$, where $| \pm\rangle=\frac{1}{\sqrt{2}}(|0\rangle \pm|1\rangle)$. For a given graph state, there are many closest product states that lead to the same exact value of the entanglement, and they are equivalent up to local operation, and we give one form as the representation.

For the graph states with entanglement 3.9275, the form of the closest product state is

$$
|\phi\rangle=|+\rangle^{\otimes 3}\left|\phi_{4}\right\rangle^{\otimes 2}\left|\phi_{1}\right\rangle^{\otimes 3}|0\rangle
$$

For the graph states with entanglement 4.9275 , the form of the closest product state is

$$
|\phi\rangle=|-\rangle^{\otimes 2}\left|\phi_{2}\right\rangle^{\otimes 2}|1\rangle^{\otimes 2}\left|\phi_{3}\right\rangle^{\otimes 2}\left|\phi_{2}\right\rangle
$$

As for different graph state, the sequence of the components may be different, but the numbers of the three components are identical. From the closest product state we can see that the graph states with noninteger entanglement $(\mathrm{k}+0.9275)$ indeed have ring 5 graph state as the substructure for their product states also have five $\left|\phi_{,}\right\rangle$.
TABLE II. GRAPH STATES WITH NONINTEGER ENT ANGLEMENT

\begin{tabular}{|c|c|c|c|c|c|c|c|c|c|}
\hline NO 9. & El & Eu & E & Ps & NO 9. & El & Eu & E & Ps \\
\hline 7 & 3 & 4 & 3.9275 & 0.64 & 258 & 4 & 5 & 4.9275 & 0.69 \\
27 & 4 & 5 & 4.9275 & 0.94 & 262 & 4 & 5 & 4.9275 & 0.70 \\
28 & 4 & 5 & 4.5850 & 0.95 & 267 & 4 & 5 & 4.9275 & 0.76 \\
47 & 4 & 5 & 4.9275 & 1.00 & 273 & 4 & 5 & 4.9275 & 0.72 \\
72 & 4 & 5 & 4.5850 & 0.99 & 275 & 4 & 5 & 4.9275 & 0.89 \\
79 & 3 & 4 & 3.9275 & 0.87 & 276 & 4 & 5 & 4.9275 & 0.81 \\
81 & 3 & 4 & 3.9275 & 0.90 & 277 & 4 & 5 & 4.9275 & 0.83 \\
83 & 4 & 5 & 4.9275 & 1.00 & 278 & 4 & 5 & 4.9275 & 0.88 \\
121 & 4 & 5 & 4.9275 & 0.57 & 279 & 4 & 5 & 4.9275 & 0.69 \\
125 & 4 & 5 & 4.9275 & 0.62 & 283 & 4 & 5 & 4.9275 & 0.80 \\
160 & 4 & 5 & 4.9275 & 0.95 & 284 & 4 & 5 & 4.9275 & 0.79 \\
163 & 4 & 5 & 4.9275 & 0.87 & 286 & 4 & 5 & 4.9275 & 0.76 \\
166 & 4 & 5 & 4.9275 & 0.64 & 295 & 4 & 5 & 4.9275 & 0.78 \\
167 & 4 & 5 & 4.9275 & 0.73 & 300 & 4 & 5 & 4.9275 & 0.53 \\
168 & 4 & 5 & 4.9275 & 0.85 & 304 & 4 & 5 & 4.9275 & 0.58 \\
169 & 4 & 5 & 4.9275 & 0.86 & 312 & 4 & 5 & 4.9275 & 0.78 \\
170 & 4 & 5 & 4.9275 & 0.95 & 315 & 4 & 5 & 4.9275 & 0.64 \\
188 & 4 & 5 & 4.9275 & 0.74 & 320 & 4 & 5 & 4.9275 & 0.88 \\
214 & 4 & 5 & 4.9275 & 0.74 & 340 & 4 & 5 & 4.9275 & 0.56 \\
218 & 4 & 5 & 4.9275 & 0.73 & 342 & 4 & 5 & 4.9275 & 0.81 \\
222 & 4 & 5 & 4.9275 & 0.67 & 357 & 4 & 5 & 4.9275 & 0.63 \\
227 & 4 & 5 & 4.9275 & 0.98 & 361 & 4 & 5 & 4.9275 & 0.56 \\
236 & 4 & 5 & 4.9275 & 0.86 & 364 & 4 & 5 & 5.5124 & 1.00 \\
238 & 4 & 5 & 4.9275 & 0.65 & 436 & 4 & 5 & 5.8381 & 0.02 \\
257 & 4 & 5 & 4.9275 & 0.67 & & & & & \\
\hline
\end{tabular}

$$
E_{u}=E_{\text {LOCC }}, E_{l}=E_{b i}
$$

TABLE III. GRAPH STATES WITH INTEGER ENTANGLEMENT

$4 \leq E \leq 5$

\begin{tabular}{|c|c|c|c|c|c|c|c|c|c|c|c|}
\hline NO 9. & E & Ps & NO 9. & E & Ps & NO 9. & E & Ps & NO 9. & E & Ps \\
\hline 126 & 5 & $0.61(1)$ & 333 & 5 & $0.77(1)$ & 380 & 5 & $1.00(2)$ & 412 & 5 & $0.56(1)$ \\
136 & 5 & 0.31 & 335 & 5 & $0.85(1)$ & 381 & 5 & $0.48(1)$ & 413 & 5 & 0.73 \\
150 & 5 & $0.92(1)$ & 338 & 5 & $0.97(2)$ & 382 & 5 & $0.60(1)$ & 414 & 5 & $0.81(1)$ \\
151 & 5 & $0.76(1)$ & 339 & 5 & $0.45(1)$ & 383 & 5 & 1.00 & 415 & 5 & $0.75(1)$ \\
164 & 5 & $0.99(1)$ & 344 & 5 & $0.72(1)$ & 384 & 5 & $0.63(1)$ & 416 & 5 & $0.66(1)$ \\
206 & 5 & $0.97(1)$ & 345 & 5 & $0.88(1)$ & 385 & 5 & $0.80(1)$ & 417 & 5 & 0.57 \\
212 & 5 & $0.69(2)$ & 347 & 5 & $0.85(1)$ & 386 & 5 & 0.62 & 418 & 5 & $0.42(1)$ \\
213 & 5 & $0.71(1)$ & 348 & 5 & $0.56(1)$ & 387 & 5 & $0.98(1)$ & 419 & 5 & 0.95 \\
217 & 5 & $0.81(1)$ & 349 & 5 & $0.71(1)$ & 388 & 5 & $0.73(1)$ & 420 & 5 & $0.45(1)$ \\
219 & 5 & $0.52(1)$ & 350 & 5 & $0.66(1)$ & 390 & 5 & 0.45 & 421 & 5 & $0.26(1)$ \\
259 & 5 & $0.51(1)$ & 352 & 5 & $0.72(1)$ & 392 & 5 & 0.52 & 422 & 5 & $0.54(1)$ \\
261 & 5 & $0.64(1)$ & 353 & 5 & 0.38 & 393 & 5 & $0.91(1)$ & 423 & 5 & $0.42(1)$ \\
264 & 5 & $0.78(1)$ & 355 & 5 & 0.42 & 394 & 5 & 1.00 & 424 & 5 & $0.87(1)$ \\
265 & 5 & $0.74(1)$ & 356 & 5 & $0.70(1)$ & 395 & 5 & 0.66 & 425 & 5 & $0.58(1)$ \\
269 & 5 & $0.55(1)$ & 358 & 5 & $0.58(1)$ & 396 & 5 & $0.62(1)$ & 426 & 5 & 1.00 \\
270 & 5 & $0.53(1)$ & 359 & 5 & $0.41(1)$ & 397 & 5 & $0.90(1)$ & 427 & 5 & $0.62(1)$ \\
274 & 5 & 0.57 & 360 & 5 & $0.47(1)$ & 398 & 5 & 1.00 & 428 & 5 & 0.46 \\
281 & 5 & 0.34 & 363 & 5 & $0.75(1)$ & 400 & 5 & $0.82(1)$ & 429 & 5 & 1.00 \\
297 & 5 & $0.50(1)$ & 365 & 5 & $0.85(1)$ & 401 & 5 & $0.41(1)$ & 430 & 5 & 0.86 \\
299 & 5 & $0.87(1)$ & 366 & 5 & 0.50 & 402 & 5 & $0.45(1)$ & 431 & 5 & 1.00 \\
302 & 5 & $0.80(1)$ & 368 & 5 & $1.00(2)$ & 404 & 5 & $0.42(1)$ & 432 & 5 & 0.28 \\
305 & 5 & $0.85(1)$ & 371 & 5 & $0.73(2)$ & 405 & 5 & 0.48 & 433 & 5 & 0.53 \\
307 & 5 & $0.65(1)$ & 372 & 5 & 0.95 & 406 & 5 & 0.51 & 434 & 5 & 0.81 \\
310 & 5 & $0.57(1)$ & 375 & 5 & $0.82(1)$ & 407 & 5 & $0.55(1)$ & 435 & 5 & 0.82 \\
311 & 5 & 0.42 & 376 & 5 & $0.46(1)$ & 408 & 5 & 0.91 & 437 & 5 & 0.84 \\
318 & 5 & 0.36 & 377 & 5 & $0.97(2)$ & 409 & 5 & 0.96 & 438 & 5 & 0.95 \\
331 & 5 & $0.85(2)$ & 378 & 5 & 1.00 & 410 & 5 & $0.70(1)$ & 439 & 5 & $0.85(1)$ \\
332 & 5 & $0.99(2)$ & 379 & 5 & $0.86(1)$ & 411 & 5 & $0.78(1)$ & 440 & 5 & 0.96 \\
\hline
\end{tabular}


The next graph state set with noninteger entanglement $(\mathrm{k}+0.5850)$ is specified by [[6, 0, 4]] stabilizer state [12][17], the entanglement of the stabilizer sate is

$$
E=2+\log _{2} 3 \approx 3.5850
$$

The closest product state is

$$
|\phi\rangle=\left|\phi_{3}\right\rangle^{\otimes 3}\left|\phi_{4}\right\rangle^{\otimes 3}
$$

For the graph states with entanglement 4.5850 , the form of the closest product state is

$$
|\phi\rangle=|+\rangle^{\otimes 2}\left|\phi_{3}\right\rangle^{\otimes 5}|0\rangle\left|\phi_{1}\right\rangle
$$

It also has $\operatorname{six}\left|\phi_{j}\right\rangle$.

There are two special graph states whose bounds of entanglement are $4 \leq E \leq 6$, i.e. No 9.364 and No 9.436 .

The entanglement of No 9.364 graph state is 5.5124, the form of the closest product state is

$$
|\phi\rangle=\left|\phi_{4}\right\rangle^{\otimes 2}\left|\phi_{2}\right\rangle^{\otimes 5}\left|\phi_{4}\right\rangle^{\otimes 2}
$$

The entanglement of No 9.436 graph state can't be exactly determined, the value of entanglement maybe 5.8381, but the successful probability of iteration is very low, which need for further research.

There are some graph states whose entanglements are integer 5, and the components of the closest product states contain $|0\rangle$, where $|0\rangle=\frac{1}{2}(|0\rangle-i|1\rangle)$. The forms of the closest product state are

$$
|\phi\rangle=|O\rangle|+\rangle^{\otimes 2}|0\rangle^{\otimes 4}|O\rangle^{\otimes 2}
$$

or

$$
|\phi\rangle=|1\rangle^{\otimes 2}|+\rangle^{\otimes 4}|1\rangle^{\otimes 3}
$$

As for different graph states, the numbers of components $|0\rangle,| \pm\rangle,|0\rangle$ or $|1\rangle$ maybe different.

In summary, the closest product states of graph states with noninteger entanglement have indispensable component $\left|\phi_{j}\right\rangle$, but for graph states with integer entanglement, the closest product states do not contain $\left|\phi_{j}\right\rangle$, but $|\mathrm{O}\rangle$.

For some special graph states, direct application of iterative algorithm fails, the entanglement and closest product states for these graph states can also be obtained, but the iteration algorithm should be modified, we explain in the next section.

\section{PRECISION OF ITERATION}

In order to verify the effectiveness of iterative algorithm, we concentrate on the precision of iteration for calculating the entanglement of graph states whose lower and upper bounds do not meet. Let $\Delta=\left|E_{\text {numeric }}-E_{\text {theory }}\right|$ be the computational error of the iteration, where $E_{\text {numeric }}$ is the entanglement determined by iteration, $E_{\text {theory }}$ is the exact value of entanglement [16] proposed in the former section. We give the successful probabilities of achieving the precision within $\Delta \leq 10^{-14}$ for some reasonable rounds of iteration with random initial conditions. From the actual numerical calculations, we can see that a precision of $10^{-14}$ is limited by the computer for our iterative algorithm.

For all graph states presented in Table II and Table III, the entanglement of a part of them can be calculated by applying algorithm directly, and the successful probabilities $\left(P_{s}\right)$ are listed in Table II and Table III. The round of the iteration is set to 150 , in order to calculate the successful probability, we run the algorithm 100 times for each graph state to count the number of algorithm that achieves the precision $\Delta \leq 10^{-14}$.

For the other part of graph states, direct application of iterative algorithm fails, and the values of entanglement calculated by iteration are all greater than the upper bounds or uncertain. The reason is that the iterative equations (7) are correlated [12], thus to obtain the maximal fidelity, we should omit some of the equations and use these remain equations for iteration. In calculation, we set one or two $z_{j}$ to random numbers that do not change in the iteration. Since we don't know the exact number of equations that are correlated, we calculate all possible choices of fixing $z_{j}$. The successful probabilities are shown in Table II and Table III, notation (1) behind the successful probabilities indicates that we omit one of the equations. After a series of calculations, the successful probabilities shown in Table II and III are the best.

After modifying the iterative equations, the closest product states can also be obtained. The only difference is that if we omit an iterative equation, there will be two arbitrary components in the closest product states, and omit two, there will be four arbitrary components. That is because we set one or two $z_{j}$ to random numbers.

For the graph states with entanglement 4.9275, omitting one iterative equation, such as No 9.27, 9.150, the form of the closest product states is

$$
|\phi\rangle=|-\rangle \forall\left|\phi_{3}\right\rangle^{\otimes 3}\left|\phi_{2}\right\rangle \forall\left|\phi_{2}\right\rangle|1\rangle
$$

Where $\forall$ is an arbitrary component.

For the graph states with entanglement 5 , such as omitting one iterative equation, the forms of the closest product states are

$$
|\phi\rangle=|-\rangle^{\otimes 2}|1\rangle^{\otimes 2}|-\rangle \forall|1\rangle \forall|1\rangle
$$




$$
|\phi\rangle=\forall|O\rangle^{\otimes 2}|+\rangle|O\rangle^{\otimes 4} \forall
$$

For the graph states with entanglement 5, such as omitting two iterative equations, the form of the closest product states is

$$
|\phi\rangle=\forall|+\rangle \forall^{\otimes 2}|+\rangle \forall|1\rangle^{\otimes 3}
$$

In summary, for the graph states whose entanglement calculated by modified iterative equations, the structural characteristics of closest product state remain the same, the only difference is containing arbitrary components which are reasonable. For graph states with noninteger entanglement, the closest product states contain $\left|\phi_{j}\right\rangle$, as for integer entanglement graph states, the closest product states contain $|\mathrm{O}\rangle$.

\section{CONCLUSIONS}

The entanglement of 9-qubit graph states can be determined by two steps. First, we confirm the upper and lower bounds, if the upper and lower bound equal, then the entanglement is the bound; second, if the two bounds unequal, we need adopt iteration algorithm to find the closest product state, then calculate the entanglement. The precision of the iteration algorithm can be less than $10^{-14}$. For some special graph states the iteration algorithm can not be directly applied, because the iterative equations may correlate with each other, in order to calculate the entanglement, we should omit one or two iterative equations. The entanglement values for the 9-qubit graph states maybe integer or noninteger, the feature of the closest product states to the graph states with noninteger entanglement is that it has $\left|\phi_{j}\right\rangle$ as the indispensable components, for graph states with integer entanglement, the closest product states do not contain $\left|\phi_{j}\right\rangle$, but $|\mathrm{O}\rangle$. For a given graph state, there are many local equivalent closest product states, they all lead to the same exact value of the entanglement. In all the cases of unequal bounds, the entanglement may be equal to its upper bound (integer) or between the bounds (noninteger). 9-qubit graph state appears two new structures comparing to 8-qubit graph state, one can be determined, but the other need further research.

\section{ACKNOWLEDGMENT}

We are grateful for the funding of the National Natural Science Foundation of China (Grant No. 60972071), Zhejiang Province Science and Technology Project (Grant No.2009C31060).

\section{REFERENCES}

[1] R. Raussendorf, D. E. Browne, and H. J. Briegel, Phys.Rev. A 68, 022312 (2003).

[2] D.-M. Schlingemann, Stabilizer codes can be realized asgraph codes, Quant. Inf. Comp. 2, 307 (2002)

[3] D.-M. Schlingemann, Cluster states, graphs and algorithms,Quant. Inf. Comp. 4, 287 (2004), quantph/0305170

[4] M. Grassl, A. Klappenecker, and M. R*otteler, Graphs,Quadratic Forms, and Quantum Codes, in Proc. 2002IEEE International Symposium on Information Theory,Lausanne, Switzerland (2002), page 45.

[5] J. H. Bevis, G. S. Domke, and V. A. Miller, Ranks of trees and grid graphs, J. of Combinatorial Math. andCombinatorial Computing 18 (1995), 109-119.

[6] G. Vidal and R. Tarrach, Phys. Rev. A 59, 141(1999).

[7] V. Vedral, M. B. Plenio, M. A. Rippin, P. L. Knight, Phys.Rev.Lett. 78, 2275(1997).

[8] V. Vedral and M. B. Plenio, Phys. Rev. A 57, 1619(1998).

[9] T-C. Wei, P. M. Goldbart, Phys.Rev.A, 68, 042307(2003).

[10] M. Hayashi, D. Markham, M. Murao, M. Owari and S.Virmani, Phys. Rev. A, 77, 012104 (2008)

[11] D. Markham, A. Miyake, and S. Virmani, New. J. Phys. 9, 194(2007).

[12] X. Y. Chen, eprint, arXiv: 0906.1603V2[quant-ph]

[13] M. Hein, W. Dur, J. Eisert, R. Raussendorf, M. van den Nest and H. J. Briegel, eprint, arXiv: quant-ph/0602096.

[14] McKay, B. D.: nauty User's Guide. 2003. http://cs.anu.edu.au/ bdm/ nauty/nug.pdf.

[15] L. E. Danielsen. http://www.ii.uib.no/ larsed/vncorbits/

[16] X. Y. Chen, eprint, arXiv: 0906.5130[quant-ph]

[17] M. Hein, J. Eisert, and H. J. Briegel, arXiv: 03071307130v7[quant-ph] 